\title{
A method for phylogenetic reconstruction of aneuploid cancers based on multiregional genotyping data
}

Natalie Andersson ( $\square$ natalie.andersson@med.lu.se )

Lund University https://orcid.org/0000-0002-3643-4404

\section{Subhayan Chattopadhyay}

Lund University

Anders Valind

Lund University

Jenny Karlsson

Lund University

David Gisselsson

Lund University https://orcid.org/0000-0002-0301-426X

\section{Article}

Keywords: tumor heterogeneity, pediatric cancer, phylogenetic reconstruction

Posted Date: January 22nd, 2021

DOl: https://doi.org/10.21203/rs.3.rs-140537/v1

License: (9) This work is licensed under a Creative Commons Attribution 4.0 International License. Read Full License

Version of Record: A version of this preprint was published at Communications Biology on September 20th, 2021. See the published version at https://doi.org/10.1038/s42003-021-02637-6. 


\section{A method for phylogenetic reconstruction of aneuploid cancers based on multiregional genotyping data}

Natalie Andersson ${ }^{1}$, Subhayan Chattopadhyay ${ }^{1}$, Anders Valind $^{1,2}$, Jenny Karlsson ${ }^{1}$ \& David Gisselsson ${ }^{1,3,4}$

${ }^{1}$ Division of Clinical Genetics, Department of Laboratory Medicine, Lund University, Lund, Sweden.

${ }^{2}$ Department of Pediatrics, Skåne University Hospital, Lund, Sweden. ${ }^{3}$ Division of Oncology-Pathology, Department of Clinical Sciences, Lund University, Lund, Sweden. ${ }^{4}$ Clinical Genetics and Pathology, Laboratory Medicine, Lund University Hospital, Skåne Healthcare Region.

Correspondence and request for materials should be addressed to N.A. (email: natalie.andersson@med.lu.se).

Abstract word count: 129

Text word count: 6563

References: 37

Figures: 4

Tables: 1

Supplementary Figures: 7

Supplementary Datasets: 17 


\section{Abstract}

2 Phylogenetic reconstruction of cancer cell populations remains challenging. There is a particular lack of

3 tools that deconvolve clones based on copy number aberration analyses of multiple tumor biopsies

4 separated in time and space from the same patient. This has hampered investigations of tumors rich in

5 aneuploidy but few point mutations, as in many childhood cancers. Here, we present DEVOLUTION,

6 an algorithm for subclonal deconvolution followed by phylogenetic reconstruction from bulk

7 genotyping data. It integrates copy number and sequencing information across multiple tumor regions

8 throughout the inference process, provided that the mutated clone fraction for each mutation is known.

9 We validate DEVOLUTION on SNP-array data from 56 pediatric tumors comprising 253 tumor

10 biopsies confirming concordance to biological mechanisms and show a robust performance on extensive

11 simulations of bulk genotyping data.

12

13

14

15

16

17

18

19

20

21 


\section{Introduction}

Neoplasms are a heterogenous group of diseases driven by Darwinian selection. Most cancers are presumed to originate from a single mutated cell, from which each mutation is conveyed to its daughter cells, that in turn can acquire additional aberrations, establishing subpopulations (subclones) of cells with diverse genetic compositions within the tumor ${ }^{1}$. This evolution of cancer cells is further shaped by genetic drift and selection pressure from the tumor microenvironment and oncological treatment ${ }^{2-5}$. Due to this process, many cancers exhibit vast intratumor heterogeneity (ITH) as well as intertumor heterogeneity between the primary tumor and its metastases ${ }^{6-10}$. Knowledge about how ITH emerges over time remains limited and multiple models have been proposed to explain it such as punctuated, neutral, linear, and branched evolution as well as a big bang model of tumor growth followed by neutral evolution $^{11-13}$. By analyzing the genetic variation of the tumor spatially as well as temporally, mathematical methods can be employed in order to reconstruct its evolution, commonly in the form of a phylogenetic tree that links together distinct cancer cell subpopulations in an inferred temporal order. Such phylogenetic reconstructions can improve the understanding of tumorigenesis, progression to metastatic disease, and aid the development of novel therapeutic strategies ${ }^{7,8,14}$.

One of the biggest challenges in phylogenetic analysis of bulk sample data from tumors is that the genetic analysis of each sample is conducted on millions of cells at once, usually constituting multiple subclones. The relative proportions of the subclones within each biopsy may also vary across the biopsied regions, stressing the need to integrate information from multiple biopsies separated in space to thoroughly assess the genetic profile of the tumor. Not addressing this may result in the prediction of illicit biological trajectories and so-called biopsy trees, not constituting true phylogenies ${ }^{15}$. Phylogenetic relationships should thus ideally be constructed based on the deconvolved clonal structure, i.e. one should infer which subclones are characterized by which alterations and, in addition, the ancestral order of these.

Even though single cell sequencing (SCS) has emerged as an important tool for temporal reconstruction that circumvents the issue of clonal deconvolution it is very costly to implement on the scales needed in 
the clinic and usually provides limited sequence coverage ${ }^{16}$. A more cost-effective alternative is to 51 perform computerized deconvolution of bulk genotyping data derived from single nucleotide polymorphism array (SNP-array), targeted deep sequencing (TDS), whole exome sequencing (WES) or whole genome sequencing (WGS). Bulk genotyping yields a set of genetic alterations present in each biopsy along with information that can be utilized to estimate the proportion of cells harboring each aberration in each biopsy, denoted the mutated clone fraction $(\mathrm{MCF})^{17}$. However, most tools developed for computerized deconvolution of bulk genotyping data focuses solely on somatic point mutations, presumes a diploid background, and lacks specific pipelines to handle intratumoral heterogeneity of copy number alterations (CNAs) of chromosomal segments or whole chromosomes. In addition, they do not provide the possibility to infer phylogenetic trees solely on copy number aberration data from multiple biopsies separated in time and space ${ }^{18-21}$. Since most cancers are aneuploid to some degree ${ }^{22}$, this is a serious shortcoming, especially for cancer types where aneuploidy is a common feature such as high-grade adult carcinomas and many childhood cancers ${ }^{8,23}$. Consequently, there is a particular need for tools capable to infer phylogenetic trees based on multiregional copy number data.

To fill this methodological gap, we introduce DEVOLUTION, an algorithm for subclonal deconvolution followed by phylogenetic reconstruction from bulk genome profiles including high-resolution copy number data (e.g. from SNP-array, WES or WGS) and sequencing information (e.g. from WES, WGS or TDS) separately or in unison. The deconvolution is based on à priori MCF-estimation of the individual aberrations in each sample and the algorithm systematically combines information from all available biopsies throughout the inference process to reconcile the most probable temporal evolution of the tumor by inferring an event matrix that is used to reconstruct phylogenetic trees. Importantly it can deduce evolutionary trajectories based on copy number data alone. In addition, predictions of the subclonal size and compositions across biopsies are visualized directly in the phylogenetic tree. DEVOLUTION provides an objective framework for creating event matrices and phylogenetic trees 74 from bulk genotyping data, avoiding subjective bias compromising the validity of tree-to-tree comparisons (S. Table 1). 
To demonstrate DEVOLUTION's utility, the algorithm was evaluated using SNP-array data from 253 tumor regions from 56 pediatric cancers including neuroblastoma (NB), Wilms tumor (WT), and rhabdomyosarcoma (RMS), comprising the most common extracranial, solid tumors in children. Additionally, the algorithm showed a robust performance on simulated multiregional bulk genotyping data. DEVOLUTION holds the potential to facilitate further insights into the development, progression, and response to treatment, particularly in tumors with high burden of chromosomal copy number alterations.

\section{Results}

\section{Overview of the algorithm workflow}

The algorithm operates on multiregional sampling data analyzed using whole genome profiling followed by MCF-computation (Figure 1a-c). The input file is an $u \times v$ dimensional matrix, containing information about the $u$ genetic alterations detected in a tumor. For each alteration there are $v$ columns indicating the genetic position, alteration type as well as the proportion of cells in the biopsy harboring the alteration (Supplementary Figure 1). For copy number aberrations, the matrix is subjected to an algorithm identifying all unique events across the samples while considering the uncertainty in the aberration breakpoint measurement (Supplementary Figure 2). The DBSCAN (density-based spatial clustering of applications with noise) algorithm is then used to identify clusters of genetic alterations having similar cellular proportions across multiple samples, indicating that they might be reflecting a group of cells having an identical genetic profile (Figure 1d). By identifying these clusters of genetic alterations, the computational load can be decreased and the unfolding of the subclonal composition aided.

DBSCAN prepares the data set to be subjected to the subclonal deconvolution algorithm, which is 
101 biopsies is integrated throughout this process to minimize the occurrence of parallel evolution (PLC)

102 and back mutation contradictions (BMC). PLC in copy number data means that the same type of genetic

103 alteration with the same genomic start- and endpoints in the chromosome appears independently in

104 different cells within the tumor. This is, in most cases, unlikely from a biological standpoint, unless

105 there is a copy number alteration including an entire chromosome. BMC incorporates genetic alterations

106 that are gained and then lost further down the evolutionary history in the tree, which may be feasible

107 scenarios for some types of genetic alterations such as a gain of a whole chromosome that is later lost

108 but are less likely to occur for structural chromosomal aberrations and point mutations, and should never

109 be occur for loss of heterozygosity events ${ }^{24}$.

110 In addition, the user can provide a matrix containing information about illicit orders of genetic events,

111 that can be taken into consideration during the deconvolution (Figure 1e). The deconvolution

112 culminates in a suggestion of the most likely temporal order of all genetic aberrations, constituting the

113 basis for the creation of an event matrix, illustrating the distribution of genetic alterations across

114 subclones (Figure 1f). Using this event matrix, the biological distance between the subclones is

115 calculated using the Hamming distance ${ }^{25}$ and phylogenetic trees are reconstructed using the maximum

116 likelihood and parsimony methods. In addition, the algorithm provides the distribution and size of the

117 clusters across the samples, resulting in an overview of the dynamics, spatial distribution, and

118 dissemination of the tumor (Figure 1g).

120 Validation on pediatric tumors confirms concordance to biological mechanisms

121 DEVOLUTION was applied to a previously reported dataset of 56 pediatric cancers and phylogenetic

122 trees were generated based on copy number data for 22 neuroblastomas, 20 Wilms tumors and 8 123 rhabdomyosarcomas comprising a total of 253 biopsies (Figure 2, Supplementary Figure 3,

124 Supplementary File 1). Event matrices but not phylogenetic trees could be reconstructed from six 125 patients (NB1, NB24, WT1, WT2, WT3 and WT5) in which all samples from the same patient had 126 identical genomic profiles. 
127 The phylogenetic trees of the remaining 50 tumors all represented plausible biological scenarios and

128 often illustrated key events in tumor evolution. For example, in NB5 (Figure 2a), where a primary tumor 129 and a metastasis presented at the same time, the metastasis was demonstrated to originate from a 130 population of cells having the genetic alterations of the stem as well as one group of cells encompassing 131 a subclone also present in the primary tumor. This indicates polyclonal seeding. A more complex pattern 132 of polyclonal seeding was observed in NB22 (Figure 2b), a patient with progressive tumor growth 133 across multiple metastatic locations. Here there was more subclonal variation among the lymph node 134 metastasis than among metastases to distant organs. The metastases to distant organs often presented as 135 solitary branches, exemplified by the same subclone colonizing both the lung and skull. This might 136 indicate that the threshold for tumor cells to escape the primary tumor and colonize the lymph nodes is 137 lower than for colonization of distant organs, displayed as a wider variety of different subclones across 138 lymph node locations compared to an extensive selection for a certain subclone in distant loci. WT11

139 and WT19 (Figure 2c and d) both showed subclones that were distributed across several locations 140 within the primary tumors, a phenomenon which has previously been demonstrated to be common in 141 Wilms tumors ${ }^{17}$. RMS8, an alveolar rhabdomyosarcoma (Figure 2e), displayed an intricate evolutionary 142 pattern with many genetic alterations. Here the primary tumour's subclones form a cluster at the root of 143 the tree, while the cell populations from a metastasis and a local relapse share a branch having a vast 144 amount of additional genetic alterations. Hence, phylogenetic trees produced from copy number profiles 145 by DEVOLUTION, can provide biological insights that might aid the understanding of how cancer 146 develops and progresses in individual patients.

\section{Contradictions are rarely seen in the phylogenetic trees}

149 In $80 \%$ of all tumors analyzed, the maximum likelihood (ML) and parsimony (MP) methods resulted 150 in identical phylogenetic trees (19/22 NB, 17/20 WT, 4/8 RMS) (Figure 3a-d). When the ML and MP 151 tree for the same case did differ from one another, the differences in the branching structure were minor

152 (Supplementary Figure 3). We identified the positions of the genetic copy number alterations in each 
153 tree to identify contradictions based on prior knowledge about how genetic aberrations occur in cancer 154 cells. More specifically, we analyzed instances of PLC and BMC.

155 When the ML and MP trees differed from one another, this was always due to a PLC/BMC in the 156 phylogeny, often with both contradictions together in the same tree. PLC and/or BMC were found in 157 14/50 ML-trees and 14/50 MP-trees (5 NB, 5 WT, 4 RMS), hence 28/100 trees in total. Of these, 3/5

$158 \mathrm{NB}, 3 / 5 \mathrm{WT}$ and 1/4 RMS trees contained only one single contradiction located among the leaves of the 159 trees i.e. it did not have any significant impact on the tree structure. In addition, there did not seem to

160 be any apparent difference between the frequency of the types of contradictions between ML and MP 161 trees (Figure 3e). Excluding the cases with PLC and BMC of whole chromosomes, which are plausible 162 events, only 8/50 ML-trees and 8/50 MP-trees (0 NB, 4 WT and 4 RMS) exhibited contradictions in the 163 tree structure. In these eight cases, merely a few genetic alterations caused the PLC and/or BMC. They 164 were caused by aberrations altering clone size compared to another event across samples or similar 165 aberrations that still fell outside the breakpoint cutoff for similarity causing them to be considered 166 separate events. These situations may be resolvable by critically reviewing the original data 167 (Supplementary Figure 4). Alternating clone sizes was particularly common in the RMS trees 168 (Supplementary Figure 5). The RMS tumors had a significantly higher total branch length than NB 169 and WT (Figure 3b), indicating a more complex genomic profile. They also had a mean number of 170 genetic alterations per biopsy of 20 , most of them present in $>50 \%$ of cells in a single biopsy thus 171 allowing just one single solution of the temporal evolution. To prompt review of original data when 172 pertinent, the software will warn the user that there is a contradiction in the data set and the tree might 173 therefore not be entirely biologically accurate.

\section{Evaluation using simulated data}

176 The reliability of the algorithm was further evaluated using simulated bulk sampling data. In the patient 177 data set, the median number of unique subclonal alterations in total across all biopsies were 6 for NB, 5 178 for WT and 13 for RMS. To accommodate this large variation, the simulation was conducted for three 

40000 virtual tumor cells (Figure 4a). Virtual biopsies were sampled randomly from the set of cells

181 while varying the number of biopsies from 1 to 10 , generating a segment file along with a list of true 182 unique subclones across biopsies for each mutational frequency. Hence, analysis could be performed using DEVOLUTION while having the true subclonal composition at hand.

As expected, when increasing the number of biopsies or the mutation frequency more genetic aberrations were identified. In addition, a higher number of subclones were correctly allocated. (Figure 4b). A higher number of overall mutations will provide the software with more information, which is why the number of correctly allocated genetic alterations increase with mutation frequency for the same number of biopsies. Specifically, the number of positions in the temporal sequence at which genetic alterations can be allocated decreases with the number of genetic alterations. The number of unique subclones increases while the proportion of cells in each biopsy representing each subclone decreases. However, when more alterations are correctly allocated in absolute numbers, the proportion of correctly allocated alterations will not. Throughout the different mutational frequencies $93 \pm 5.5 \%$ of the genetic

193 alterations were correctly allocated in the event matrix (Figure 4c). Thus, when increasing the number

194 of biopsies, the absolute number of correctly allocated genetic alterations increases, but the proportion 195 of correctly allocated alterations does not change significantly. The reason for this is that sampling 196 additional locations will also increase the chance of finding an area with a late genetic alteration that is 197 hard to correctly place in the phylogeny because of its low spatial dissemination. We further dissected 198 why not all subclones were correctly allocated. It was hypothesized to occur due to low spatial 199 dissemination, resulting in the presence of certain genetic alterations in a subset of biopsies. Excluding 200 all genetic alterations found in only one single biopsy in fact resulted in the correct allocation if $99.5 \pm$ $2010.8 \%$ of the genetic alterations in this mixture of entities (Figure 4d-e). 


\section{Relationship to existing work}

206 Much progress has been made in the field of clonal deconvolution. Many methods are although limited 207 to integrating information from a single biopsy, such as TITAN and THetA in addition to only accepting 208 sequence data ${ }^{26,27}$. PyClone and SciClone are, however, employable on multiple biopsies, but assume 209 that all detected CNAs are clonal i.e., present in all cells, do not infer the evolutionary relationship 210 between the identified clusters and sequence data is required for them to operate ${ }^{19,20}$. In addition, 211 SciClone focuses exclusively on SNVs in copy number neutral and loss of heterozygosity (LOH) free 212 portions of the genome. Both PyClone and SciClone mainly operate as clustering algorithms and do not 213 infer the order of genetic alterations, hence their output could be used as an input to DEVOLUTION. 214 Hence, they fulfill different purposes and is not meant to exclude one other. PhyloWGS on the other 215 hand can use both SNAs and CNAs to infer a phylogeny and is employable on multiple samples. The 216 algorithm does although not integrate information between samples during the inference procedure, 217 representing a loss valuable information, and is limited to WGS data ${ }^{18}$. Also, other methods such as 218 Clomial, LiCHeE and SCHISM are specifically designed for SNAs and cannot solely include CNAs to 219 infer a phylogeny ${ }^{28-30}$. REVOLVER also requires sequencing data, cannot use CNA-data alone and is 220 specifically designed to integrate phylogenies from large cohorts of patients in order to infer common 221 trajectories of repeated evolution. SPRUCE requires input of sequencing data to infer phylogenetic trees.

222 Hence, most methods available focus exclusively on sequencing data and there is currently no available 223 tool that can reconstruct a phylogenetic tree based on multiregional SNP-array data alone, which is a commonly used genotyping method in the clinic. Additionally, many methods focused on SNA only 225 outputs clusters of genetic alterations and their MCF. Event matrices are subsequently often constructed 226 manually from these MCF estimates, posing a risk for unintentional subjective bias in the deconvolution 227 process, especially when integrating information from multiple biopsies conjointly. These methodological gaps are filled by DEVOLUTION, which provides a subjective method to infer phylogenies based on a priori MCF estimations based on preset rules that are employed equally across all patient data, hence providing a standardized framework for inferring phylogenies from bulk genotyping data, thus allowing tree-to-tree comparison without the risk of bias from subjective curation. 


\section{Discussion}

236 A single biopsy from a tumor can consist of multiple distinct subclones and their prevalence may vary

237 across biopsied areas. Not addressing this fact when studying cancer cell evolution can be deleterious

238 and result in incorrect phylogenies ${ }^{15}$, stressing the need for multispatial and temporal sampling to unfold

239 the genomic landscape. DEVOLUTION thoroughly assesses the problem by combining information

240 obtained across multiple biopsied regions throughout the entire subclonal deconvolution effectively

241 deconvolving subclones transversing clonal territories, with the potential to concomitantly include both

242 point mutations and copy number alteration data. In contrast to other methods DEVOLUTION allows

243 phylogenetic trees to be constructed using copy number information alone and integrating information

244 from multiple biopsied areas throughout the inference procedure. The algorithm can combine data from

245 SNP-array, TDS, WES and WGS, provided the MCF for each genetic alteration is known, which can be

246 computed based on the $\log 2$ ratio for copy number alterations or variant allele frequencies (VAF) for

247 point mutations as described extensively elsewhere ${ }^{17,19,20}$. Since aneuploidy is a common feature across

248 many adult carcinomas and a majority of childhood cancers, the integration of copy number aberrations

249 in the phylogeny holds the potential to increase the understanding of the evolution of these diseases. If

250 needed, there is also a possibility for user curation, for example if it is known that certain genetic

251 alterations cannot co-exist.

252 Evaluating the software using high-throughput SNP-array data from 253 multitemporal and spatial 253 regions from 56 pediatric tumors produced phylogenetic trees that were well in concordance with prior 254 knowledge of how chromosomal aberrations occur in cancer cells. Surprisingly, generating phylogenetic 255 trees using ML and MP predominantly yielded identical tree structures. When thoroughly examining 256 these trees, contradictions such as PLC and BMC were identified in the cases where the ML/MP trees 257 differed, which were found to always be due to disagreements in the original data set. The user is 
therefore encouraged to reevaluate this genetic alteration in the input segment file, since it may be

259 particularly subjected to noise or keep the tree if the phylogenetic situation is considered biologically

260 plausible. The extensive evaluation of the two methods did not indicate that the choice of mathematical

261 method favors a certain type of error in the phylogenetic tree.

262 Clustering genetic alterations using DBSCANs were sufficient for analyzing the pediatric tumors 263 incorporated in this study and the simulated data sets. The size of $\epsilon$ can be changed by the user to 264 increase the flexibility in the clustering, thus optimizing it further for the data set at hand to account for 265 noise. Note that the purpose of the ad hoc clustering mainly is to reduce the computational complexity. 266 If the clustering is too strict, the DEVOLUTION algorithm compensates by thoroughly integrating the 267 information across samples. The clustering algorithm can easily be changed.

268 In summary, we have seen how DEVOLUTION can be used to analyze the intratumoral heterogeneity 269 as well as the intertumoral heterogeneity between the primary tumor and metastasis through evaluation 270 on a dataset of pediatric tumors harboring extensive aneuploidy. By analyzing a cancer's phylogenetic 271 tree, an overview of its heterogeneity and temporal order of genetic alterations can be assessed, which can be used to follow the tumor's evolutionary response to treatment ${ }^{31,32}$ and may aid the identification 273 of subclones posing a risk of metastasizing, relapsing or being resistant to therapy. It may also make it 274 possible to identify genetic alterations that seems to appear early in the tumor's development, posing 275 attractive targets for therapy since they are present in a large proportion or all cells in the tumor.

\section{Conclusions}

278 DEVOLUTION produces phylogenetic trees from multiregional cancer genomics data, integrating copy 279 number aberrations and sequence mutations separately or in unison, provided the MCF is known. The 280 algorithm reliably deconvolves subclones and infers the evolutionary history in well in concordance to 281 prior knowledge of how genetic aberrations occur in cancer cells. It performs robustly across real data 282 from multiple aneuploid tumor types and in simulated data across a wide variation in mutation rates and sample numbers. DEVOLUTION holds the potential to facilitate insights into the development, 

number alterations.

\section{Methods}

\section{The software}

The major structure of the software can be divided into five steps

1. Preprocessing of the data.

2. Clustering of genetic alterations based on information from multiregional sampling from the same patient.

3. Subclonal deconvolution based on information from multiregional sampling from the same patient.

4. Construction of an event matrix.

5. Usage of a mathematical model to reconstruct the phylogenetic trees, in this case

\section{Preprocessing of the data}

The input data consist of an $u \times v$ dimensional matrix containing information about the $u$ detected genetic alterations present in each biopsy. The matrix should also specify the genetic location of each alteration, its type (gain, loss, cnni etc.) as well as the proportion of cells harboring the alteration in that particular biopsy (the mutated clone fraction, MCF), represented by the $v$ columns (Supplementary

Figure 1). How the MCF can be computed from $\log 2$ ratios as well as VAFs is described extensively elsewhere ${ }^{17}$. Also, methods such as PyClone and SciClone could be used to infer clusters of somatic mutations and their corresponding VAFs across samples, which could then be used to calculate the MCF. 
If allelic copy number alterations are considered, the user is advised to choose a cutoff for the detected

310 genetic alterations in the segment file to be considered separate events, reflecting the measurement 311 uncertainty regarding the start and end positions of the genetic alterations. The default cutoff is $1 \mathrm{Mbp}$.

312 The user can also choose which data types to include in the analysis. In this way e.g. SNP array and sequencing data can be analyzed separately for comparison or in unison without having to separate the matrix manually.

The algorithm scans the MCFs for missing values, indicating that the MCF has not been able to be determined. If the event is considered to belong to the stem, based on biological knowledge or additional data, the missing value is replaced by $100 \%$. Amplicon accumulation is an example of such a case when it is not possible to determine the fraction of cells harboring it since the number is hypervariable. A stem event is defined as the presence of the alteration in $\geq 90 \%$ of the cells in all samples. The alterations containing missing values for MCF are removed entirely if part of a subclone to not overestimate genetic variation within the tumor.

The clustering algorithm was constructed to localize all unique genetic alterations throughout the tumor samples. The program loops through the rows of the data file representing the genetic aberrations. For each row it compares the genetic alterations and their position on the chromosome to all the other rows, representing other detected genetic aberrations throughout the samples. If the events' start or end positions differ by a certain cutoff, set by the user based on the measurement uncertainty of the data set, and/or they are different aberration types, they are considered as two separate events, else they are considered as the same event (Supplementary Figure 2). Thus, all conditions stated below must be met for the algorithm to consider two alterations detected in the same patient to be the same.

1. Alteration 1 and 2 are localized on the same chromosome.

2. Alteration 1 and 2 harbor the same type of alteration.

3. Neither alteration 1 nor 2 should belong to the stem.

a. Alterations belonging to the stem are always considered as separate events.

4. $\mathrm{X}_{1}=\left\|\mathrm{A}_{1_{\text {start }}}-\mathrm{A}_{2_{\text {start }}}\right\| \leq \mathrm{co}_{\mathrm{ev}}$ 
5. $\mathrm{X}_{2}=\left\|\mathrm{A}_{1_{\text {end }}}-\mathrm{A}_{2_{\text {end }}}\right\| \leq \mathrm{co}_{\mathrm{ev}}$

336 In the present study, considering allelic copy number aberrations, the cutoff $\left(\mathrm{co}_{\mathrm{ev}}\right)$ for measurement uncertainty in the start and end position of the events was set to $1 \mathrm{Mbp}$, also constituting the default for DEVOLUTION. Since the chromosome sizes ranges from 48-250 Mbp this cutoff constitutes a start and end point deviation of $0.4-2 \%$ of the chromosome length.

Clustering of genetic alterations incorporating information from multiregional and temporal sampling from the same patient

343 In our model a tumor is proposed to consist of multiple subpopulations of cells that harbor different sets of genetic alterations. Each individual alteration is part of a mutation space $m_{i} \in\left\{m_{1}, m_{2} \ldots m_{\theta}\right\}$ comprising all mutations present in the tumor where $i, \theta \in \mathbb{N}^{+}$and $\theta$ is the total number of mutations. The mutational profile obtained from the biopsies thus represent a subset of the total mutation space and is the information at hand to describe the evolutionary trajectory of the tumor. For this purpose, all detected mutations are combined with their respective MCF-values into a matrix representing their distribution across samples (Supplementary Figure 6). For a particular tumor, this results in a matrix $\mathrm{T}_{\mathrm{MxB}}$ with the dimensions $\mathrm{M} \times \mathrm{B}$, where $\mathrm{M}$ is the total number of unique genetic alterations and $\mathrm{B}$ is the total number of biopsies available. Hence $m_{\delta}$ indicates a certain genetic alteration $\delta$, and $b_{\omega}$ represent a biopsy $\omega$. The value $t_{\delta \omega}$ consequently corresponds to the MCF for an alteration, $m_{\delta}$, in a sample, $b_{\omega}$ where $t_{\delta \omega} \in[0,100]$ i.e. it is bound between 0 and $100 \%$. This can be written as

$$
\mathrm{T}_{\mathrm{MxB}}=\left[\begin{array}{cccc}
\mathrm{t}_{1,1} & \mathrm{t}_{1,2} & \ldots & \mathrm{t}_{1, \mathrm{~B}} \\
\mathrm{t}_{2,1} & \mathrm{t}_{2,2} & \ldots & \mathrm{t}_{2, \mathrm{~B}} \\
\vdots & \vdots & \ddots & \vdots \\
\mathrm{t}_{\mathrm{M}, 1} & \mathrm{t}_{\mathrm{M}, 2} & \ldots & \mathrm{t}_{\mathrm{MB}}
\end{array}\right]
$$

$$
\text { where } \mathrm{t}_{\delta \omega} \in[0,100], \delta \in\{1, \ldots, \mathrm{M}\}, \omega \in\{1, \ldots, \mathrm{B}\} \text { and } \delta, \omega \in \mathbb{N}^{+}
$$

In order to generate phylogenetic trees illustrating the relationship between the subclones present in the tumor, which aberrations reside in the same cells as well as which subpopulations of cells the tumor consist of must be determined. To solve this, the idea is that a true subclone of cells should form a cluster 
of unique genetic alterations that persist. They should remain grouped irrespective of inclusion of new

360 data from an additional region of the primary tumor or metastasis. Alterations that seem to follow each

361 other are more likely to be in the same cells. The first step is thus to yield a clustering to identify groups

362 of genetic alterations, uniquely identifying a certain subclone. The subsequent step is to determine the temporal order of the alterations in question, since each subclone will represent a linear combination of the clusters identified. Note that for DEVOLUTION the clustering is only used to reduce the computational complexity for the upcoming subclonal deconvolution algorithm. Mostly alterations are clustered that show similar MCF in all available biopsies. This is not to be confused with the more intricate clustering methods used in for example SciClone.

Density based clustering techniques such as DBSCAN ${ }^{33}$ are superior at unsupervised clustering of nonuniform clusters. Furthermore, the number of clusters does not have to be specified beforehand, which you have to do with many other established clustering algorithms. In addition, it does only have two hyperparameters named $\operatorname{minPts}$, which is the minimal number of points that is allowed in a cluster, and $\epsilon$ representing the radius in which points i.e. the genetic alterations' position in the B-dimensional space,

373 are included, where B is the total number of biopsies. If $\epsilon$ is chosen too small a large part of the data 374 might not be clustered and choosing it too big will put all alterations in the same cluster. The choice of $375 \epsilon$ can be aided by using a k-distance-graph which illustrates the distance to the minPts- $1=\mathrm{k}$ nearest 376 neighbor. The value to choose is when this plot shows an elbow which can be obtained by visual 377 inspection. Another method would be to create a vector between the start- and endpoint of the graph. 378 Then create a vector perpendicular to this having its start position at this line and its end position at our data curve. The elbow can be computed by finding the vector with the largest magnitude. The clustering

380 method is not integrated directly in the subclonal deconvolution algorithm described below and is 381 therefore easy to replace or alter if needed for noisier data ${ }^{33}$.

382 The algorithm provides a matrix containing all clusters of genetic alterations. Let $\mathrm{C}_{\mathrm{KxN}}$ be the matrix 383 representing the clusters of genetic alterations. It has the dimensions $\mathrm{K} \times \mathrm{N}$ where $\mathrm{K}$ is the number of 384 genetic alterations in the cluster and $\mathrm{N}$ is the cluster number. All matrix positions $\mathrm{c}_{\mathrm{kn}} \neq 0$ are unique 385 i.e. the same genetic alteration cannot belong to multiple clusters. 


$$
\mathrm{C}_{\mathrm{KxN}}=\left[\begin{array}{cccc}
\mathrm{c}_{1,1} & \mathrm{c}_{1,2} & \cdots & \mathrm{c}_{1, \mathrm{~N}} \\
\mathrm{c}_{2,1} & \mathrm{c}_{2,2} & \cdots & \mathrm{c}_{2, \mathrm{~N}} \\
\vdots & \vdots & \ddots & \vdots \\
\mathrm{c}_{\mathrm{K}, 1} & \mathrm{c}_{\mathrm{K}, 2} & \cdots & \mathrm{c}_{\mathrm{KN}}
\end{array}\right]
$$

where $c_{\mathrm{kn}} \in m_{\delta} \wedge c_{k n} \neq c_{e d},\left(\forall k, e \in\{1, \ldots, K\} \& n, d \in\{1, \ldots, N\} \wedge c_{k n} \neq 0\right)$

A matrix representing the clusters present in each biopsy and their size determined by the mean of the aberrations in the cluster is also constructed.

$$
\mathrm{Z}_{\mathrm{CxB}}=\left[\begin{array}{cccc}
\mathrm{z}_{1,1} & \mathrm{z}_{1,2} & \cdots & \mathrm{z}_{1, \mathrm{~B}} \\
\mathrm{z}_{2,1} & \mathrm{z}_{2,2} & \cdots & \mathrm{z}_{2, \mathrm{~B}} \\
\vdots & \vdots & \ddots & \vdots \\
\mathrm{z}_{\mathrm{C}, 1} & \mathrm{z}_{\mathrm{C}, 2} & \cdots & \mathrm{z}_{\mathrm{CB}}
\end{array}\right] \text { where } \mathrm{z}_{\mathrm{cb}} \in[0,100]
$$

Where $\mathrm{c}$ is a specific cluster of aberrations, $\mathrm{b}$ the biopsy and $z_{c b}$ the size of the cluster $\mathrm{c}$ in sample $\mathrm{b}$.

\section{Subclonal deconvolution based on information from multiple samples from the same patient}

394 The space of a single biopsy is $100 \%$ and the space of all biopsies can thus be represented by a matrix where $\mathrm{p}$ is the partitioning of the available space in the biopsy and $\mathrm{s}_{\mathrm{pb}}$ is the space available in a specific partitioning $\mathrm{p}$ in biopsy $\mathrm{b}$. Initially $\mathrm{s}_{1, \mathrm{~b}}=100 \wedge \mathrm{s}_{\mathrm{p} \neq 1, \mathrm{~b}}=0$.

$$
\mathrm{S}_{\mathrm{PxB}}=\left[\begin{array}{cccc}
\mathrm{s}_{1,1} & \mathrm{~s}_{1,2} & \ldots & \mathrm{s}_{1, \mathrm{~B}} \\
\mathrm{~s}_{2,1} & \mathrm{~s}_{2,2} & \ldots & \mathrm{s}_{2, \mathrm{~B}} \\
\vdots & \vdots & \ddots & \vdots \\
\mathrm{s}_{\mathrm{P}, 1} & \mathrm{~s}_{\mathrm{P}, 2} & \ldots & \mathrm{s}_{\mathrm{PB}}
\end{array}\right] \text { where } s_{p b} \in[0,100] \text { and } \sum_{\mathrm{p}=1}^{\mathrm{P}} s_{p, b}=100 \wedge b \in\{1, \ldots, \mathrm{B}\} \in \mathbb{N}^{+}
$$

The clusters of aberrations in each biopsy, as supplied by $\mathrm{Z}_{\mathrm{CxB}}$, are allocated to the space in decreasing order, altering the magnitude of the spaces in $\mathrm{S}_{\mathrm{PxB}}$ based on the MCF of the clusters allocated to it.

The allocation iteration algorithm is initially conducted considering each sample individually, resulting in a matrix encompassing all possible allocations of the clusters in every biopsy. Subsequently, all possible allocations throughout the samples are addressed to minimize the occurrence of parallel evolution. The algorithm hence tries to produce one uniform solution of the temporal order of events that does not contradict any information provided in the biopsies. The solution should be in concordance to every biopsy provided. If not possible, parallel evolution or back mutations will occur in the final 
phylogenetic tree and the user is advised to reconsider the original data set, since it may be biologically unlikely. It may be possible to allocate a cluster to multiple positions without producing contradicting 408 temporal orders in any of the samples, for which the largest available space assumption is employed to make an objective decision, based on the presumption that the mutational frequency is equal in all cells within the biopsy no matter how many mutations they have. The cluster will consequently be placed as 411 a descendant to the cluster constituting the largest proportion of the biopsy, in the absence of further 412 biological information steering it elsewhere. Clusters presenting with only one possible allocation in a 413 biopsy provides especially valuable information concerning the temporal order of events, e.g. having a 414 group of alterations that are all present in all cells in a biopsy clearly indicates that there exists a group 415 of cells in the tumor harboring all of these alterations, aiding the temporal allocation in other samples where these alterations may present themselves as subclonal. DEVOLUTION does allow some overlap 417 in cellular frequency in the allocation algorithm taking into consideration the measurement uncertainty 418 of MCF. This iterative computation results in the subclones present in the biopsies along with an 419 estimation of their size and distribution across the samples.

\section{Incorporating user-controlled rules for avoiding imposition of illicit biological trajectories}

422 Some genetic aberrations present in the data set might be known to never occur in the same cell for some well-known biological reason. Such constraints should optimally be supplied to the algorithm to ensure biologically plausible solutions. The user can therefore provide the DEVOLUTION algorithm with a matrix indicating which genetic aberrations in the data set that cannot be placed after one another. The 426 first column represents a mother genetic alteration that the daughter alteration specified in the second column, cannot have (Supplementary Figure 7). The subclonal deconvolution algorithm extracts a list 428 for each genetic alteration containing information about in how many of the samples it can be allocated 429 after a certain cluster. There might be multiple possible solutions, equally prevalent. In this instance the 430 matrix containing information about illicit biological orders can aid the program in taking a decision 431 regarding which of these allocations are less likely, subsequently discarding them. These rules will thus 432 only be employed if the data set allows the genetic alterations to be placed in any other way. If the only 
433 possible way for the events to be allocated is to place them as descendants, the user will be advised to

434 revise the original data set. No such rules were integrated in the analysis of the 56 pediatric tumors in 435 the present study.

\section{Construction of an event matrix}

438 Based on the estimated subclonal composition, an event matrix $E=\left[\hat{a}_{1}, \hat{a}_{2} \ldots \hat{a}_{k}\right]$ was constructed 439 illustrating the distribution of genetic alterations across the identified subclones. Each $\hat{a}_{i}$ is binary vector 440 belonging to subclone $i$. Each row represents a genetic alteration indicated with a 1 if present or 0 if 441 absent in the subclone. The event matrix is used as the foundation for phylogenetic tree generation, 442 illustrating the relationship between the subclones within the tumor.

\section{Reconstruction of phylogenetic trees}

445 In order to generate the phylogenetic trees, the genetic distances between all the subclones must be 446 computed. Here, the Hamming distance was used to assess a distance matrix displaying the genetic 447 distance between each of the subclones. It computes the distance between two vectors by adding all 448 positions in which they differ from one another. Note that it does not consider the magnitude of the 449 entity in the bins compared, which does not matter in this case since they are binary, hence the branch 450 lengths in the phylogenetic trees will be in units of number of aberrations. No bias in the estimation of 451 branch lengths were seen for ML in this study.

452 Using the function stem, a column is added in which the tree will be rooted. The stem can be chosen as 453 a cell containing all mutations shared between the subclones or a cell containing no alterations thus 454 representing a normal cell. The event matrix is then transformed into phyDat format using the function 455 phydatevent. This is the data class needed for phylogenetic analysis using the $\mathrm{R}$ package phangorn ${ }^{34}$.

456 In the next step the maximum likelihood and maximum parsimony algorithms were used in order to 457 reconstruct phylogenetic trees based on the event matrices. The maximum likelihood trees were 
reconstructed using the pml algorithm in the package phangorn ${ }^{34}$. First a Hamming distance matrix was

459

calculated from the event matrix which was used to obtain an initial tree given by the neighbor joining method. The initial tree as well as the initial event matrix was used as input variables in the pml algorithm. This function returns an object containing the tree parameters, the data as well as the likelihood for that phylogenetic tree. In order to optimize the tree parameters further, the function optim.pml was used in combination with the Jukes Cantor model. The tree was subsequently rooted in a constructed cell having all the events shared between all subclones. Since the model used is time reversible the choice of the root does not influence the computed likelihood ${ }^{35}$. The tree was visualized using the ggtree package in $\mathrm{R}^{36}$.

The maximum parsimony trees were constructed using the parsimony ratchet algorithm (pratchet) in the $\mathrm{R}$ package phangorn with the Fitch algorithm ${ }^{37}$. Using the acctran algorithm the branch lengths and the ancestral character probability distributions were obtained. The trees were rooted in a cell containing no alterations.

\section{Performance testing using simulated bulk sampling data}

In order to further assess the reliability of the algorithm, it was evaluated using simulated bulk genotyping data using a basic 3D-lattice based, stochastic model of tumor growth. The simulation is initiated imagining a cell having one single genetic aberration, representing a stem event, which will be conveyed to all cells comprising the virtual tumor. In each time unit one cell can proliferate. When a cell has been chosen for proliferation a certain inherent mutation frequency determines whether the cell will mutate or not. If not, two cells identical to the mother cell are obtained, otherwise a stochastic genetic copy number aberration algorithm is used to randomly select a genetic alteration (copy number alteration), conjointly considering the chromosome boundaries and sizes. Making use of a random number generator, a chromosome is randomly selected, then a start and end position and finally the type of event. The result is one cell identical to the mother and one cell harboring one additional genetic aberration. The spatial orientation of the cells is also considered where each cell is assumed to have 26 
neighbors and two cells cannot occupy the same position. The position of the second daughter cell is randomly selected among the available neighbor positions. The simulation was conducted generating 40,000 cells giving a simple 3D lattice structure illustrating the spatial intratumoral heterogeneity for three different mutation frequencies (Figure 4a). Note that only the fractions of cells harboring a certain genetic alteration is of importance in this model and not any absolute numbers. The goal is not to correctly simulate tumor growth but to obtain a random mixture of entities to be demixed using DEVOLUTION. The mutation frequency chosen, 10/40,000, 50/40,000 and 100/40,000, is not biologically accurate but chosen such that a certain number of mutations will appear during the 492 simulation, resulting in segment files resembling the MCF-distributions seen in the patient cases. from 1 to 10. The biopsies were drawn randomly from different parts of the simulated entities. Values for $\mathrm{x}, \mathrm{y}$ and the $\mathrm{z}$ coordinates were randomly chosen while fulfilling

$$
x \in\left[x_{\min }, x_{\max }\right], y \in\left[y_{\min }, y_{\max }\right], z \in\left[z_{\min }, z_{\max }\right]
$$

where

$$
\sqrt{x^{2}+y^{2}+z^{2}} \in\left[\frac{r_{\text {mean }}}{2}, r_{\text {mean }}\right] \in \mathbb{R}
$$

Based on the position chosen, the entities within a radius of 2 units were extracted and the fraction of

501 cells harboring each of the alterations found was calculated. The data from each cell can be used to 502 create artificial bulk sampling with MCFs as well as single cell data. Hence, the segment files can be 503 analyzed while having the true subclones at hand for comparison. DEVOLUTION was evaluated using 504 1-10 biopsies for the three tumors. 
506 For phylogenetic tree characteristics, significance was tested using the Mann-Whitney U-test (two507 tailed). The stem lengths, total branch lengths and number of branches between individual patients are 508 assumed to be independent of each other and not normal distributed.

509 All simulation points were repeated at least three times and the standard deviation are illustrated with 510 bars in Figure $4 \mathrm{c}$ and $\mathrm{d}$.

\section{Data availability}

512 All data generated or analyzed during this study are included in this article as supplementary files 1-4.

\section{Code availability}

514 The code is freely available and relies on R 4.0.2 or later. Setup instructions and dependencies can be 515 found on github.

\section{Author contributions}

519 N.A. and D.G. conceived and designed the project, N.A., D.G. and S.C. developed the methodology, 520 A.V., J.K. and D.G. did tumor biopsy data acquisition, N.A. and D.G. analyzed and interpreted the data, 521 N.A., D.G., S.C., A.V. and J.K. contributed towards the manuscript.

\section{Competing interests}

523 The authors declare no competing interests.

\section{Acknowledgements}

525 We would like to thank the Swegene Centre for Integrative Biology at Lund University (SCIBLU) for 526 assistance.

\section{References}


5302 Quail, D. F. \& Joyce, J. A. Microenvironmental regulation of tumor progression and metastasis. $531 \quad$ Nat Med 19, 1423-1437, doi:10.1038/nm.3394 (2013).

5323 Komarova, N. L., Burger, J. A. \& Wodarz, D. Evolution of ibrutinib resistance in chronic 533 lymphocytic leukemia (CLL). Proc Natl Acad Sci U S A 111, 13906-13911, doi:10.1073/pnas.1409362111 (2014).

5344 Misale, S. et al. Emergence of KRAS mutations and acquired resistance to anti-EGFR therapy in 535 colorectal cancer. Nature 486, 532-536, doi:10.1038/nature11156 (2012).

5 Leder, K. et al. Fitness conferred by BCR-ABL kinase domain mutations determines the risk of pre-existing resistance in chronic myeloid leukemia. PloS one 6, e27682, doi:10.1371/journal.pone.0027682 (2011).

5396 Cresswell, G. D. et al. Intra-Tumor Genetic Heterogeneity in Wilms Tumor: Clonal Evolution and 540 Clinical Implications. EBioMedicine 9, 120-129, doi:10.1016/j.ebiom.2016.05.029 (2016).

5417 Andor, N. et al. Pan-cancer analysis of the extent and consequences of intratumor heterogeneity.

$542 \quad$ Nat Med 22, 105-113, doi:10.1038/nm.3984 (2016).

5438 Mengelbier, L. H. et al. Intratumoral genome diversity parallels progression and predicts outcome 544 in pediatric cancer. Nat Commun 6, 6125, doi:10.1038/ncomms7125 (2015).

5459 Martelotto, L. G., Ng, C. K. Y., Piscuoglio, S., Weigelt, B. \& Reis-Filho, J. S. Breast cancer intra546 tumor heterogeneity. Breast Cancer Research: BCR 16, 210, doi:10.1186/bcr3658 (2014).

$547 \quad 10 \quad$ Villamon, E. et al. Genetic instability and intratumoral heterogeneity in neuroblastoma with 548 MYCN amplification plus 11q deletion. PloS one 8, e53740, doi:10.1371/journal.pone.0053740 (2013).

$54911 \quad$ Williams, M. J., Werner, B., Barnes, C. P., Graham, T. A. \& Sottoriva, A. Identification of neutral 550 tumor evolution across cancer types. Nat Genet 48, 238-244, doi:10.1038/ng.3489 (2016).

$551 \quad 12$ Gerlinger, M. et al. Intratumor heterogeneity and branched evolution revealed by multiregion 552 sequencing. $N$ Engl J Med 366, 883-892, doi:10.1056/NEJMoa1113205 (2012).

55313 Sottoriva, A. et al. A Big Bang model of human colorectal tumor growth. Nat Genet 47, 209-216, 554 doi:10.1038/ng.3214 (2015).

55514 Andersson, N. et al. Extensive Clonal Branching Shapes the Evolutionary History of High-Risk 556 Pediatric Cancers. Cancer research 80, 1512-1523, doi:10.1158/0008-5472.CAN-19-3468 (2020).

$55715 \quad$ Alves, J. M., Prieto, T. \& Posada, D. Multiregional Tumor Trees Are Not Phylogenies. Trends 558 Cancer 3, 546-550, doi:10.1016/j.trecan.2017.06.004 (2017).

55916 Mallory, X. F., Edrisi, M., Navin, N. \& Nakhleh, L. Methods for copy number aberration detection 560 from single-cell DNA-sequencing data. Genome biology 21, 208, doi:10.1186/s13059-020-02119-8 (2020).

$561 \quad 17 \quad$ Karlsson, J. et al. Four evolutionary trajectories underlie genetic intratumoral variation in 562 childhood cancer. Nat Genet 50, 944-950, doi:10.1038/s41588-018-0131-y (2018).

56318 Deshwar, A. G. et al. PhyloWGS: reconstructing subclonal composition and evolution from 564 whole-genome sequencing of tumors. Genome biology 16, 35, doi:10.1186/s13059-015-0602-8 (2015).

56519 Roth, A. et al. PyClone: statistical inference of clonal population structure in cancer. Nat Methods 566 11, 396-398, doi:10.1038/nmeth.2883 (2014).

$56720 \quad$ Miller, C. A. et al. SciClone: inferring clonal architecture and tracking the spatial and temporal 568 patterns of tumor evolution. PLoS Comput Biol 10, e1003665, doi:10.1371/journal.pcbi.1003665 (2014). 
57122 Ben-David, U. \& Amon, A. Context is everything: aneuploidy in cancer. Nat Rev Genet 21, 44572 62, doi:10.1038/s41576-019-0171-x (2020).

$57323 \quad$ Vogelstein, B. et al. Cancer genome landscapes. Science 339, 1546-1558, 574 doi:10.1126/science.1235122(2013).

57725 Hamming, R. W. Error detecting and error correcting codes. Bell Labs Technical Journal 29, 147-

$578160(1950)$.

57926 Ha, G. et al. TITAN: inference of copy number architectures in clonal cell populations from tumor 580 whole-genome sequence data. Genome Res 24, 1881-1893, doi:10.1101/gr.180281.114 (2014). high-throughput DNA sequencing data. Genome biology 14, R80, doi:10.1186/gb-2013-14-7-r80 (2013).

$58328 \quad$ Popic, V. et al. Fast and scalable inference of multi-sample cancer lineages. Genome biology 16, 584 91, doi:10.1186/s13059-015-0647-8 (2015).

58529 Niknafs, N., Beleva-Guthrie, V., Naiman, D. Q. \& Karchin, R. SubClonal Hierarchy Inference

586 from Somatic Mutations: Automatic Reconstruction of Cancer Evolutionary Trees from Multi-region Next

587 Generation Sequencing. PLoS Comput Biol 11, e1004416, doi:10.1371/journal.pcbi.1004416 (2015).

$588 \quad 30 \quad$ Zare, H. et al. Inferring clonal composition from multiple sections of a breast cancer. PLoS 589 Comput Biol 10, e1003703, doi:10.1371/journal.pcbi.1003703 (2014).

$59031 \quad$ Maley, C. C. et al. Classifying the evolutionary and ecological features of neoplasms. Nat Rev 591 Cancer 17, 605-619, doi:10.1038/nrc.2017.69 (2017).

$59232 \quad$ Turajlic, S., Sottoriva, A., Graham, T. \& Swanton, C. Resolving genetic heterogeneity in cancer. 593 Nat Rev Genet 20, 404-416, doi:10.1038/s41576-019-0114-6 (2019).

$59433 \quad$ Ester, M., Kriegel, H.-P., Sander, J. \& Xu, X. in Proceedings of the Second International 595 Conference on Knowledge Discovery and Data Mining 226-231 (AAAI Press, Portland, Oregon, 1996).

$59634 \quad$ Schliep, K. P. phangorn: phylogenetic analysis in R. Bioinformatics 27, 592-593, 597 doi:10.1093/bioinformatics/btq706 (2011).

$598 \quad 35 \quad$ Felsenstein, J. Evolutionary trees from DNA sequences: a maximum likelihood approach. $J$ Mol 599 Evol 17, 368-376, doi:10.1007/BF01734359 (1981).

$60036 \quad$ Yu, G., Smith, D. K., Zhu, H., Guan, Y. \& Lam, T. T. Y. ggtree: an R package for visualization 601 and annotation of phylogenetic trees with their covariates and other associated data. Methods in Ecology and 602 Evolution 8, 28-36 (2017).

60337 Nixon, K. C. The parsimony ratchet, a new method for rapid parsimony analysis. Cladistics $\mathbf{1 5}$, 604 407-414 (1999). 


\section{Supplementary datasets}

609 S. Data 1 Childhood cancer data set. All segment files for NB, WT and RMS along with the clustering

610 obtained by the software. Segments are annotated similar to Supplementary Figure 1.

611 S. Data 2 The event matrices. The files named "before" illustrates the allocation within each biopsy

612 while the files denoted "after" are the final event matrices.

613 S. Data 3 Simulation results. The segment file for each simulation, the biopsy positions randomly 614 chosen, which unique cells are present in these biopsies, the event matrix and evaluation of which 615 alterations are correctly allocated using the software.

616 S. Data 4 Summary of all simulations. Tables illustrating the number of alterations in total, alterations

617 with a clone size $>10 \%$ and the number of alterations correctly allocated. 


\section{Figures}

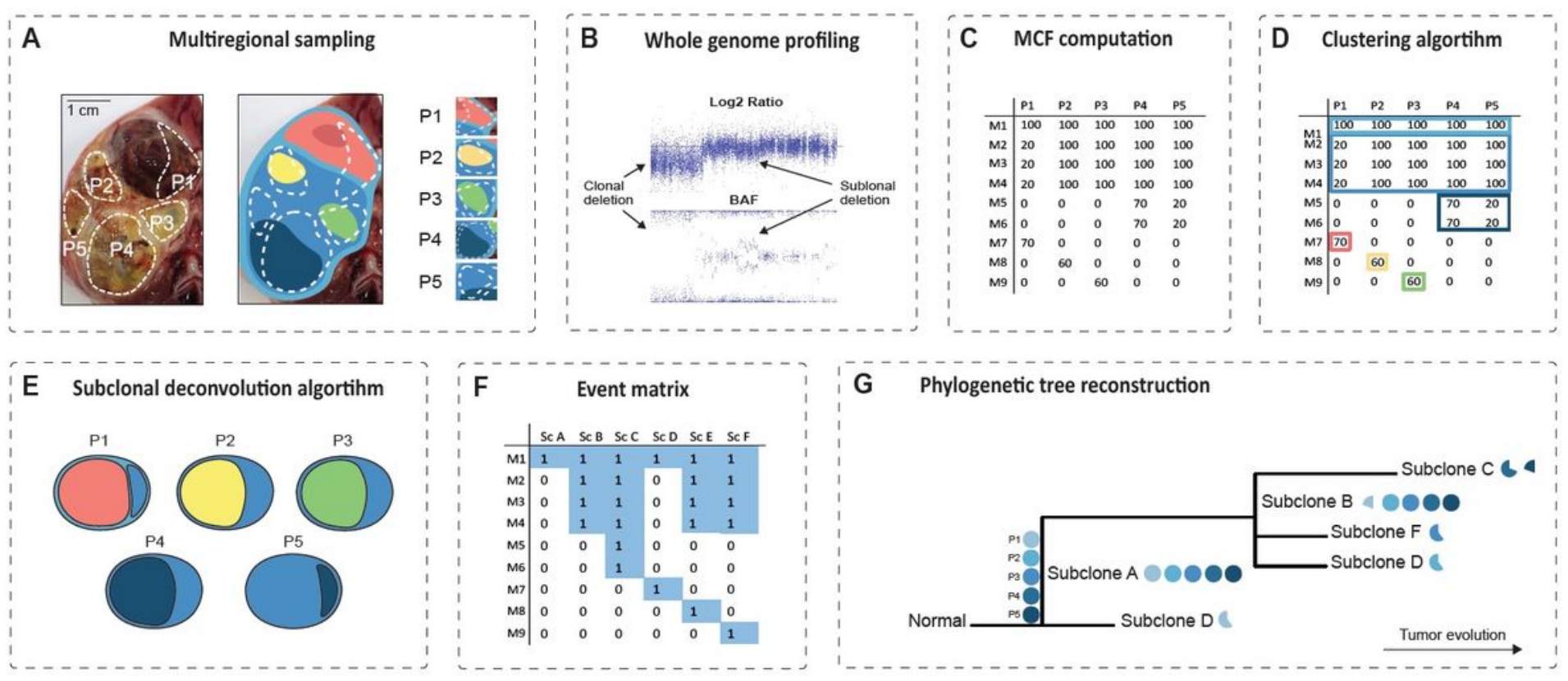

Figure 1

Overview of the methodological outline a) An example of multiregional sampling to obtain biopsies P1P5 from a Wilms tumor. The tumor is composed of several subclones with distinct genomic profiles, exemplified by the schematic genomic landscape in the rightmost panel where each color unifies cells with identical sets of genetic alterations. The photograph is adapted from a previous publication 14 and the colors do not represent true sets of genetic alterations. b) Acquisition of genomic tumor data for each sample, exemplified by copy number analysis by SNP-array. c) The whole genome profiling data can be used to compute the mutated clone fraction (MCF) illustrating the proportion of cells in each biopsy harboring a certain genetic alteration (M1-9 in the left column). d) A clustering algorithm is employed to identify genetic alterations that seem to follow each other in size across samples. e) A subclonal deconvolution algorithm determines the temporal order of these clusters by considering the information obtained throughout all samples while minimizing the occurrence of parallel evolution and back mutations. f) The proposed solution for the temporal order of subclones (Sc) is integrated into an event matrix. g) This event matrix can be used to generate phylogenetic trees with either the maximum likelihood or the parsimony method. At the stem, all available biopsies for the patient are visualized as filled circles with the biopsy name (P1-P5). At the branches of the tree the subclones can be seen along with pie charts illustrating in which biopsies, and in in what fraction of the tumor cells they appear. 
a NB5

1

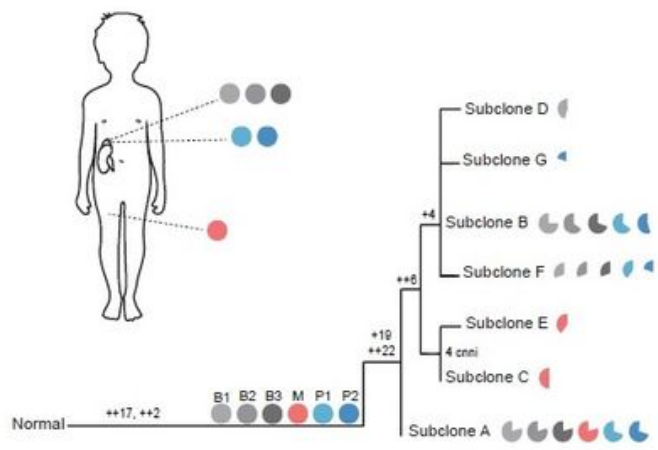

c WT11

$\stackrel{\circ}{2}$

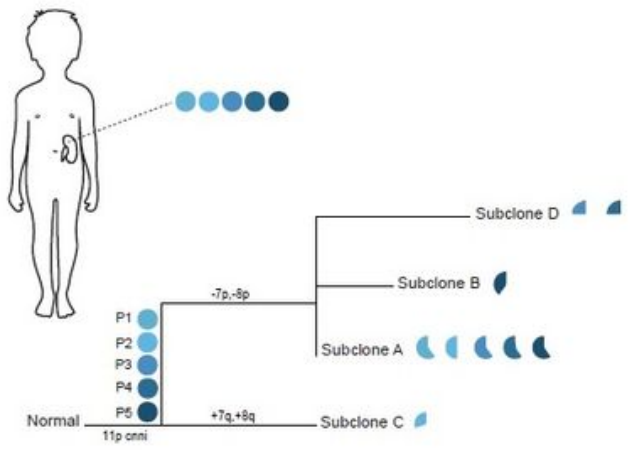

b NB22

$\stackrel{0}{\square}$

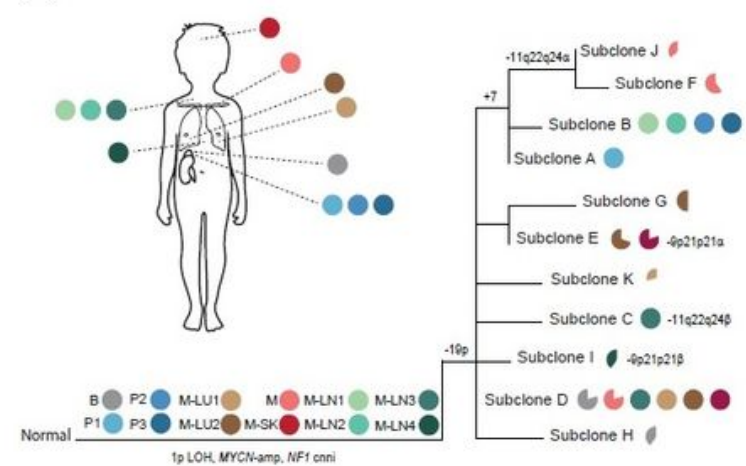

d WT19

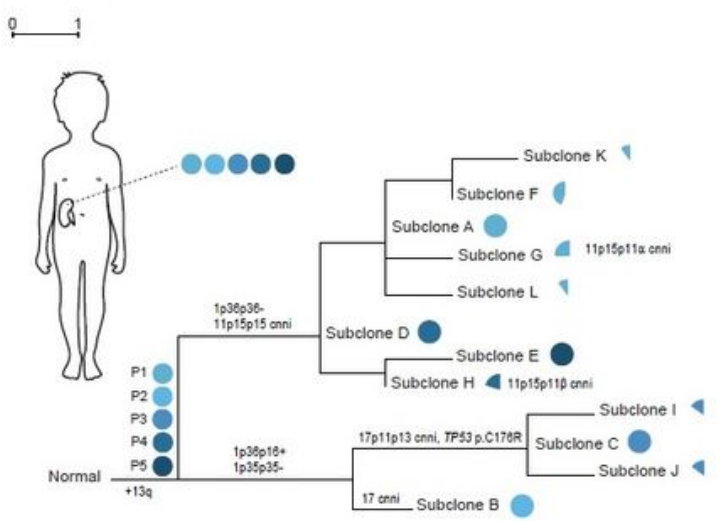

e RMS8

1

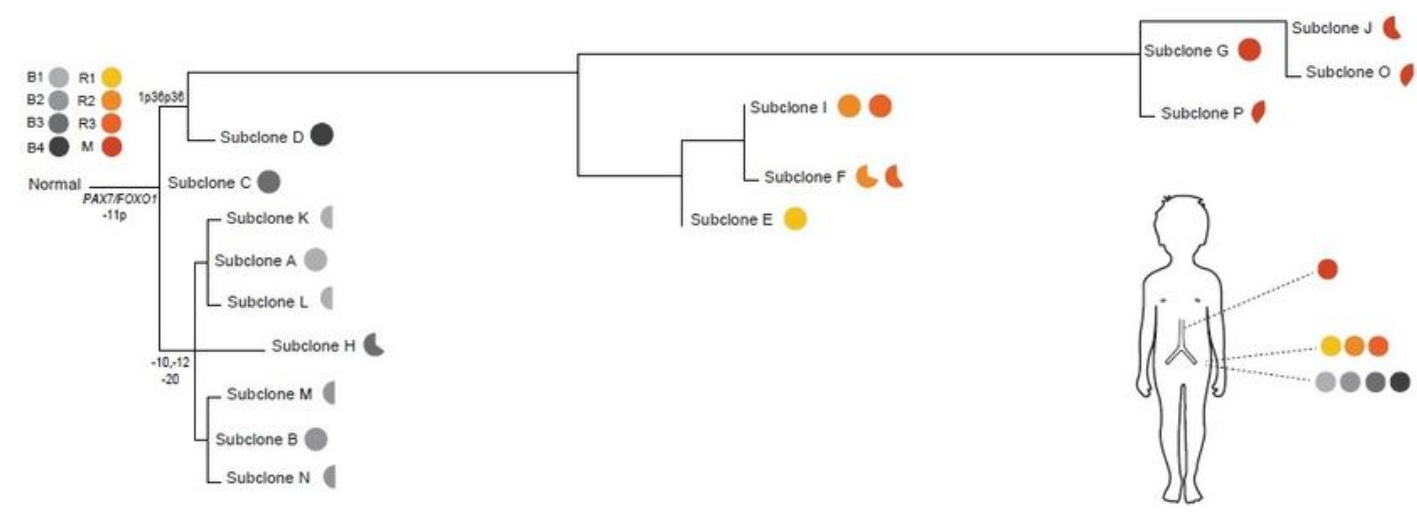

\section{Figure 2}

Phylogenetic trees of childhood cancers. At the stem of the maximum parsimony trees illustrated here, the biopsies available from each patient are denoted. The genetic alterations belonging to the stem are present in all cells in all samples, indicated by filled pies. The endpoints represent cell populations harboring distinct genomic profiles (subclones), whose fractions per sample are visualized by pie charts. The scale bar indicates the distance corresponding to one genetic aberration. Gains and losses of 
chromosomes or segments of chromosomes that are characteristic of each tumor subtype are indicated by + and - signs. a) In NB5 samples are available from the primary tumor before treatment (B1-B3), a synchronous metastasis (M), and the primary tumor post treatment (P1-P2). The metastasis must have originated from a subclone harboring the stem events only and another subclone with the copy number profile seen in subclone A, indicating polyclonal seebding. The metastasis also has a private copy number neutral imbalance (cnni) of chromosome 4. b) NB22 also shows evidence of polyclonal seeding. Samples are from the primary tumor before treatment (B), the primary tumor post treatment (P1-P3), metastases to the lung (M-LU1-2), to the lymph nodes (M-LN1-4), the skull (M-SK) and from the area around the clavicle (M). The stem harbors a 1p cnni, MYCN-amplification and a NF1 deletion. Greek letters denote different structural alterations targeting partly overlapping regions. c) WT11, shows subclones present across multiple primary tumor areas (P1-P5). d) WT19 display a similar distribution of subclones as WT11 across the post treatment

a

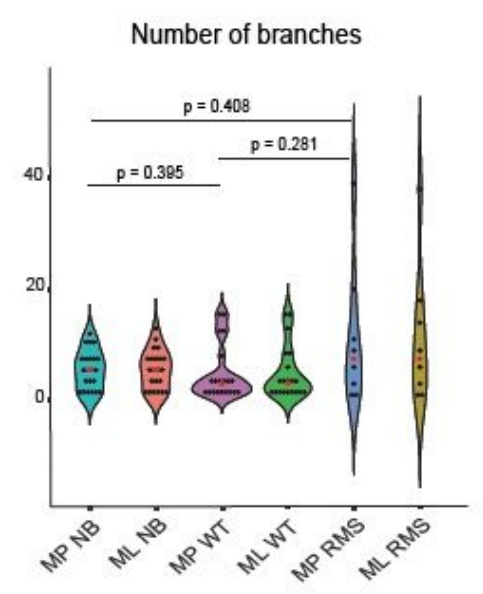

d

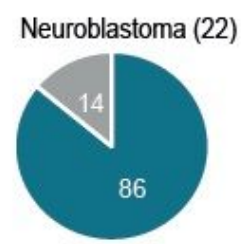

e

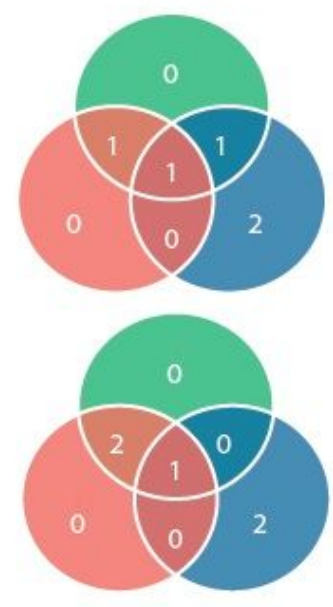

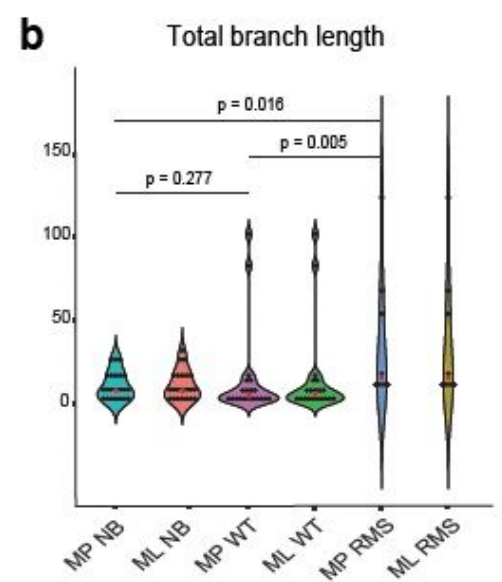

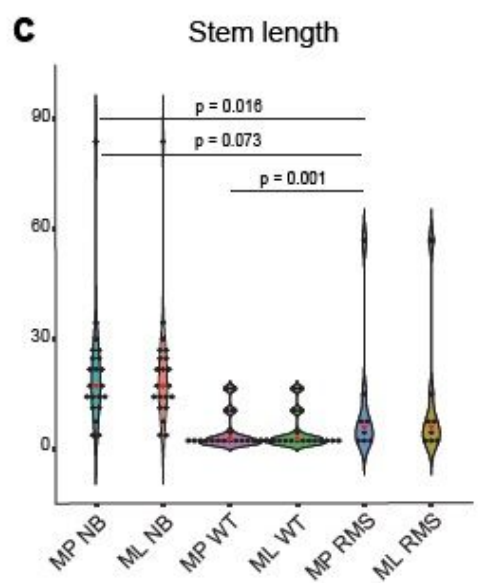

Wilms tumor (20)
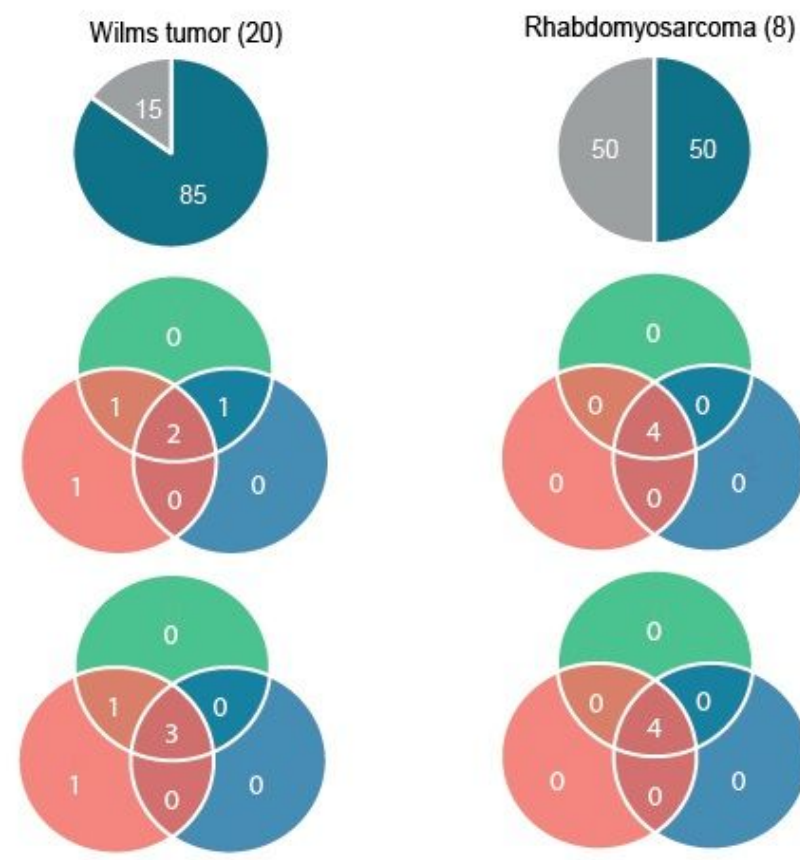

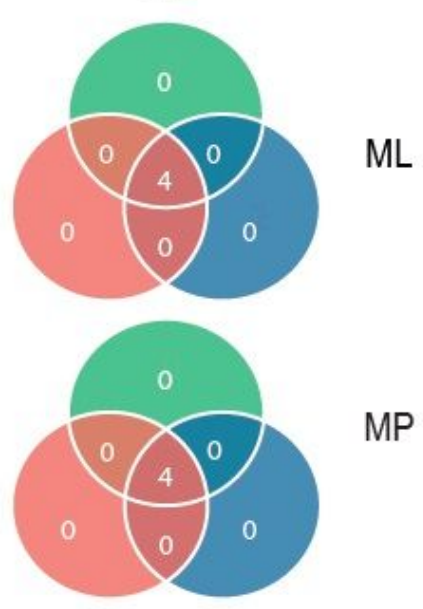

Discrepancy Identical

Parallel evolution

Back mutations

ML/MP-discrepancy

Figure 3 
Structural properties of the generated phylogenetic trees. Violin plots of a) the number of branches, b) total branch lengths and c) total stem lengths for neuroblastoma (NB), Wilms tumor (WT) and rhabdomyosarcoma (RMS) using either the maximum likelihood (ML) or parsimony (MP) method for phylogenetic reconstruction. Significance represented by P-vales were calculated using the two-sided Mann-Whitney U-test. d) In $14 \%$ of neuroblastomas, $15 \%$ of Wilms tumors and $50 \%$ of the 8 rhabdomyosarcomas analyzed, the phylogenetic trees obtained using the ML and MP methods differed from one another. NB1, NB24, WT1, WT2, WT3 and WT5 are not included in this calculation since they did not display any private genetic alterations. Hence only event matrices could be generated but not phylogenetic trees. If including these cases, the proportions would be $12.8 \%$ for NB and $12.5 \%$ for WT. e) Venn diagrams of how often discrepancies between ML/MP trees, back mutations, and parallel evolution occurred together in the same case/tree. Numbers indicate the number of tumors where a particular contradiction or combination of contradictions occurred. 


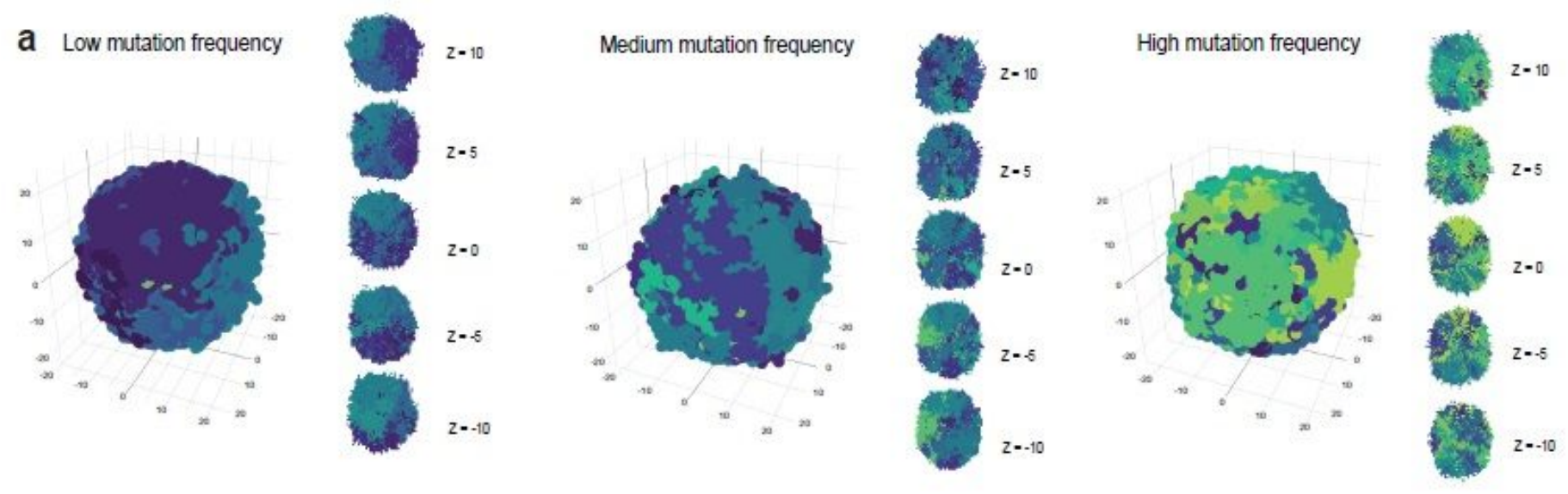

b Low mutation frequency

Medium mutation frequency

High mutation frequency

All mutation frequencies
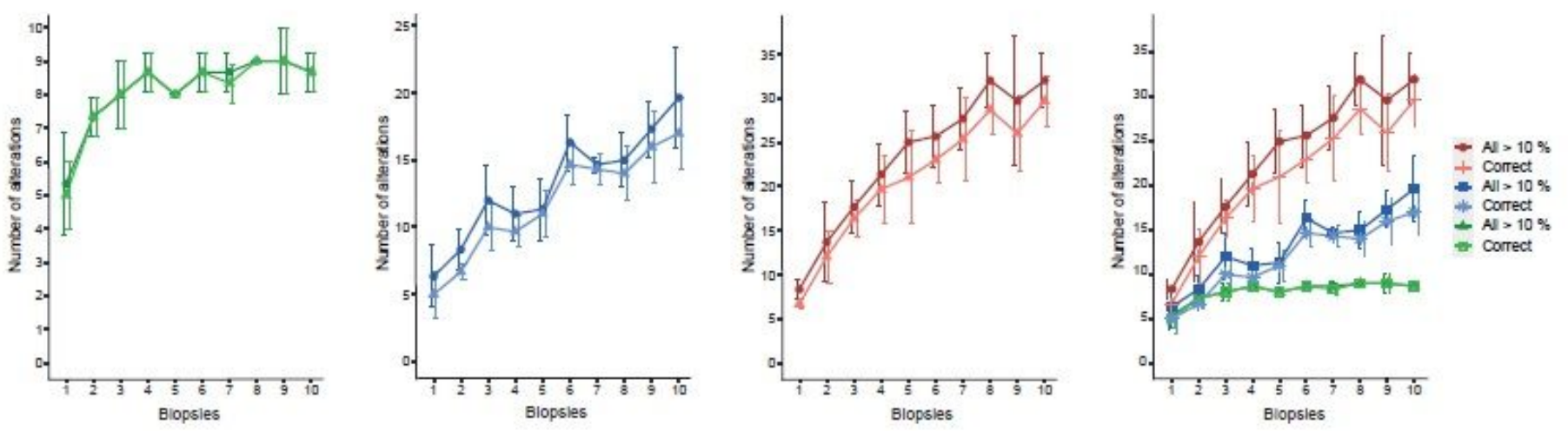

C Proportion of correctly allocated events

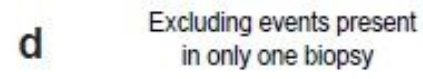

e Proportion of correctly allocated events
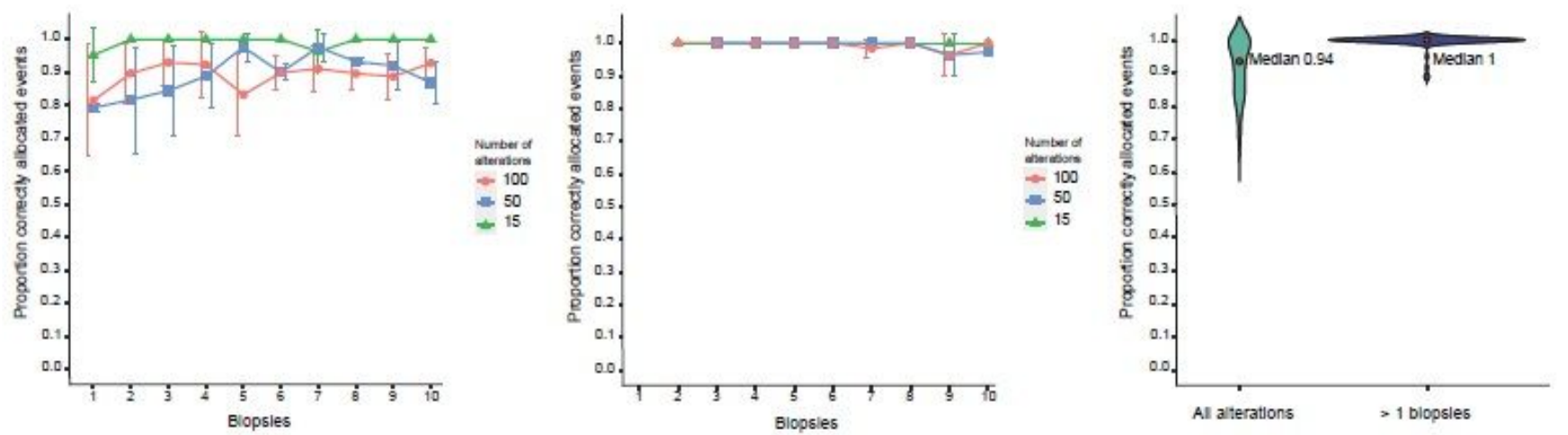

\section{Figure 4}

Properties of the simulated bulk genotyping data set and performance measure of DEVOLUTION. a) Visualization of three simulated tumors with increasing mutation frequency. To the right of each tumor are cross-sections at five positions ( $\mathrm{z}$ ). Each color represents a subclone harboring a unique genetic profile. b) When increasing the number of biopsies, more genetic alterations and hence subclones, are identified. In addition, the algorithm is also able to identify more subclones correctly. Each point in the graph is the mean of three consecutive measurements and the error bars consequently the standard deviation. c) The proportion of genetic alterations correctly allocated \pm SD when increasing the number of 
biopsies from 1-10 for three different mutation frequencies resulting in 10 (green), 50 (blue) and 100 (red) genetic alterations present in the virtual tumor. Each point is represents the mean of three consecutive measurements. d) The proportion of correctly allocated genetic alterations when excluding genetic alterations that were only found in a single biopsy. e) Violin plot showing the spread of the proportion of correctly allocated genetic alterations when including all alterations and when excluding the genetic alterations only found in one biopsy.

\section{Supplementary Files}

This is a list of supplementary files associated with this preprint. Click to download.

- S.Figure12.pdf

- S.Figure3.pdf

- S.Figure3text.pdf

- S.Figure4.pdf

- S.Figure4text.pdf

- S.Figure5.pdf

- S.Figure6.pdf

- S.Figure7.pdf 